\title{
Tuberous sclerosis complex is a novel, amyloid-independent tauopathy associated with elevated phosphorylated 3R/4R tau aggregation
}

\author{
Andy J. Liu* ${ }^{*}$, Jay B. Lusk, John Ervin, James Burke, Richard O’Brien and Shih-Hsiu J. Wang
}

\begin{abstract}
Tuberous sclerosis complex (TSC) is a neurodevelopmental disorder caused by mutations in the TSC1 and TSC2 genes and autosomal dominantly inherited. These mutations cause hyperactivation of the mammalian Target of Rapamycin (mTOR) pathway, leading to the development of nonmalignant masses involving various organ systems. Patients with TSC also experience neuropsychiatric symptoms collectively termed Tuberous Sclerosis Complex Associated Neuropsychiatric Disorder (TAND). Due to research advancements in TSC, patients now live well beyond the age of 50. Many experience objective impairment of memory and executive function, supported by formal neuropsychological testing, beginning in their late 40s. Biomarker analysis has described elevated levels of phosphorylated tau-181 in the cerebrospinal fluid of patients with TAND. Tau-PET imaging has also shown focal accumulation of the radiotracer flortaucipir (AV1451), suggesting that TSC may be a neurodegenerative disorder arising from accumulation of phosphorylated tau. However, the flortaucipir tracer has been reported to have significant off-target binding, preventing definitive conclusions from being drawn about the molecular etiology of neurodegeneration in TSC. Therefore, we initiated the Colocalization of AV1451 and Phosphorylated Tau in Adult brain tissue (CAPA) study. This study aimed to determine if flortaucipir is bound to phosphorylated tau in brains of patients with TSC and further sought to determine the specific tau isoform seen in TSC. Our results show that flortaucipir labels the 3R/4R isoform of phosphorylated tau, commonly seen in Alzheimer's disease. However, amyloid staining was negative in brains of adult patients with TSC. Therefore, we conclude that TAND symptoms are due to the accumulation of the phosphorylated tau isoform seen in Alzheimer's disease. This study suggests that hyperactivation of the mammalian Target of Rapamycin pathway may play a role in the amyloid-independent development of 3R/4R tau aggregation. Our findings could lead to a new era of anti-tau therapies used to treat both disorders.
\end{abstract}

Keywords: Tuberous Sclerosis Complex, Alzheimer's disease, Phosphorylated tau, Tauopathy, 3R tauopathy, 4R tauopathy

\section{Introduction}

Various isoforms of phosphorylated tau have been described as a neuropathological substrate for several neurodegenerative diseases. This group of disorders

*Correspondence: Andy.liu@duke.edu

Duke University, 932 Morreene Rd, Durham, NC, USA are commonly referred to as tauopathies. The $3 R$ and $4 \mathrm{R}$ isoforms of phosphorylated tau are well established [4] and are associated with various neurodegenerative conditions: the $3 \mathrm{R}$ tau is predominantly seen in Pick's disease, $4 \mathrm{R}$ tau predominantly in progressive supranuclear palsy (PSP) and corticobasal degeneration (CBD), original author(s) and the source, provide a link to the Creative Commons licence, and indicate if changes were made. The images or other third party material in this article are included in the article's Creative Commons licence, unless indicated otherwise in a credit line to the material. If material is not included in the article's Creative Commons licence and your intended use is not permitted by statutory regulation or exceeds the permitted use, you will need to obtain permission directly from the copyright holder. To view a copy of this licence, visit http://creativecommons.org/licenses/by/4.0/. The Creative Commons Public Domain Dedication waiver (http://creativeco mmons.org/publicdomain/zero/1.0/) applies to the data made available in this article, unless otherwise stated in a credit line to the data. 
and a mixture of $3 R$ and $4 R(3 R / 4 R)$ tau in Alzheimer's disease (AD).

Tuberous Sclerosis Complex (TSC) is a neurodevelopmental disorder arising primarily from mutations in TSC1 (hamartin) and TSC2 (tuberin) (TSC1/2) [8]. These mutations promote the growth of benign masses involving several organ systems. In addition to the physical manifestations of TSC, these patients frequently suffer from behavioral, cognitive and psychiatric difficulties collectively termed Tuberous Sclerosis Complex Associated Neuropsychiatric disorders (TAND) [14]. Our work published in 2019 suggested a significant overlap between TSC and a group of neurodegenerative disorders named Frontotemporal Dementias (FTD) based on neuropsychological testing, however, TSC subjects scored significantly worse on memory when compared to FTD subjects [5]. Cerebrospinal fluid (CSF) results showed elevated phosphorylated tau (pTau-181) levels with an average of $32 \mathrm{pg} / \mathrm{mL}$ in TSC subjects when compared to healthy controls [5]. Furthermore, AV1451 (Flortaucipir)-PET/ CT scans were completed in 3 adult patients with TSC. Intriguingly, focal uptake of AV1451in the frontal and temporal regions was seen in all 3 of these patients. Collectively, based on the significant memory loss and elevated CSF pTau-181 levels, it was hypothesized that TSC may represent a novel genetic tauopathy similar to that seen in $\mathrm{AD}$.

AV1451 has a high affinity for pTau-181 related to Alzheimer's disease by PET imaging [6]; however, AV1451 also displays significant off-target binding [1]. As a result, we could not definitively conclude that the focal binding of AV1451 in adult patients with TSC was due to the accumulation of pTau-181. Our current study investigates whether the AV1451 signal corresponds to tau pathology in adult patients with TSC by comparing AV1451 staining and immunohistochemical staining of various tau antibodies on brain tissue from adult patients with TSC, AD, FTLD-tau, and healthy controls. This is based on prior studies which have shown that TSC is an infantile tauopathy accumulating phosphorylated tau [10]. The isoform of phosphorylated tau in infantile tauopathies is still an area of active investigation. Here we show that AV1451 labels sparse neurofibrillary tangles in the entorhinal cortex, dorsolateral prefrontal cortex (Brodmann area 8) and inferior frontal cortex (Brodmann area 11) of adult patients with TSC. The tangles are also labeled by AT8 (which detects pTau-202/205) and GT-38 (which detects a conformation-specific mixture of 3Rand 4R-tau). Amyloid plaques were not detected in any of the brains from adult patients with TSC. These results suggest that TSC is a genetic $3 \mathrm{R} / 4 \mathrm{R}$ tauopathy that develops independent of amyloid plaques seen in AD.

\section{Materials and methods}

\section{Antibodies and PET ligand}

The antibodies used for this study were AT8 (ThermoScientific cat \# MN1020), GT-38 (ABCAM cat \# ab254274), and 4G8 (BioLegend Cat\#: 800,702). Unlabeled AV1451 was generously provided by Avid Radiopharmaceuticals.

\section{Brain tissue}

Tissue from cognitively healthy controls ( $\mathrm{HC}$ ), and from patients with AD, FTLD-tau, and TSC were obtained from the NIH biobank. Brain tissue was age and gender matched to HCs. AD brain tissue was requested with Braak stage 4 and above. Three subjects carried a clinical diagnosis of Down Syndrome (DS) and were included in the AD cohort. FTD brain tissue demonstrating FTLDtau pathology was requested. Since patients with TAND showed executive function, behavioral and memory difficulties, three brain areas involving these functions, the dorsolateral prefrontal cortex (Brodmann area 8), inferior Frontal Cortex (Brodmann area 11) and the entorhinal cortex were selected for neuropathological analysis. Formalin fixed paraffin embedded (FFPE) tissue was sectioned at eight microns on a Leica microtome.

\section{Immunohistochemistry (IHC)}

IHC was performed on formalin fixed paraffin embedded (FFPE) sections that were deparaffinized in xylene and washed in absolute ETOH and 95\% ETOH. Endogenous peroxidase activity was blocked with $1.875 \%$ $\mathrm{H}_{2} \mathrm{O}_{2}$ in methanol for $8 \mathrm{~min}$. Samples were then washed and hydrated in $\mathrm{DH}_{2} \mathrm{O}$. GT38 (ABCAM: cat\#ab254274) required antigen retrieval with a boiling citrate buffer at pH6 for $30 \mathrm{~min}$. A second blocking step was applied by submerging the sections in a dish containing $200 \mathrm{~mL}$ of $5 \% \mathrm{w} / \mathrm{v}$ nonfat dry milk in $0.05 \mathrm{M}$ Tris buffer, pH7.6, for $20 \mathrm{~min}$ at room temperature. Primary antibodies were applied to each slide (1:500 for AT8 and GT-38) and allowed to incubate for $45 \mathrm{~min}$ at $37-40^{\circ} \mathrm{C}$. Secondary antibodies were applied using the Dako EnVision ${ }^{\mathrm{TM}}$ Dual Link System-HRP (Agilent Technologies, Cat\# K4061892) and incubated for $30 \mathrm{~min}$ at $37-40^{\circ} \mathrm{C}$. The slides were then developed with Dako DAB solution (Agilent Technologies, Cat\# K346811-2) for 5 min and counterstained with Fisherfinest Hematoxylin + (ThermoFisher, Cat\#220-100) for 15-25 sec. The slides were dehydrated through graded alcohols, cleared in xylene, and coverslipped in Permount mounting medium.

\section{AV1451 staining}

The AV1451 PET Ligand is autofluorescent and was applied to FFPE sections. The sections were first deparaffinized in xylene and washed with absolute ETOH, 95\% and 70\% ETOH. Endogenous autofluorescence was 
blocked using 0.1\% Sudan Black B solution made with $70 \%$ ETOH. The AV1451 PET Ligand was supplied as a powder in a $1 \mathrm{mg}$ vial. Then $380 \mu \mathrm{L}$ of DMSO was added to the powder and allowed to dissolve to make a $10 \mathrm{mM}$ stock solution. The working solution of AV1451 was a 1:100 dilution applied for $1 \mathrm{~h}$ at room temp. The sections were rinsed in $50 \% \mathrm{ETOH}$ for $10 \mathrm{~min}$ prior to rinsing in $\mathrm{DH}_{2} \mathrm{O}$ for $10 \mathrm{~min}$. The sections were coverslipped using Vectashield Hardset(Vector Laboratories; Cat\#H1400).

\section{Imaging and data analysis}

AV1451 signal was analyzed using a digital Revolve R3 fluorescent microscope (ECHO, San Diego, CA) using the DAPI channel. AT8 and GT-38 immunohistochemistry was analyzed using an Olympus bright field microscope. Five $1 \mathrm{~mm}^{2}$ fields with the highest number of tangles were chosen from each slide. The average number of tangles was quantified and converted to a semiquantitative score as follows, 0 : no tangles, $1:<1$ tangle per $1 \mathrm{~mm}^{2}$ field, $2: 1-2$ tangles per $1 \mathrm{~mm}^{2}$ field, $3: 3-5$ tangles per $1 \mathrm{~mm}^{2}$ field, $4: 6-10$ tangles per $1 \mathrm{~mm}^{2}$ field, and 5: $>10$ tangles per $1 \mathrm{~mm}^{2}$ field.

\section{Results}

\section{Patient characteristics}

Patient characteristics are summarized in Table 1. Thirtyfive patients were included in the study, including 11 healthy control patients, 9 patients with $\mathrm{AD}, 10$ patients with TSC, and 5 patients neuropathologically diagnosed with FTLD-tau. Notably, no HC patients or patients with FTLD-tau had epilepsy, whereas $50 \%$ of patients with
TSC had epilepsy and $22 \%$ of patients with AD had epilepsy. Similarly, no control patients, patients with AD, or patients with FTD had intellectual or developmental disability (IDD), but $55 \%$ of patients with TSC had Intellectual or Developmental Disability (IDD).

\section{Qualitative immunohistochemistry findings}

Patients with TSC exhibited sparsely positive AT8 staining in the entorhinal cortex and BA8 but not BA11. AT8 staining in patients with TSC was considerably sparser than in patients with DS/AD patients or FTLD-tau. HC patients did not show appreciable AT8 staining in any region (Fig. 1). Patients with TSC exhibited more strongly positive GT38 ( $3 \mathrm{R}$ tau $+4 \mathrm{R}$ tau) staining in all three brain regions studied, although the density was still sparse compared to patients with DS/AD or FTLD-tau (Fig. 2). AV1451 staining was positive for neurofibrillary tangles in patients with TSC, FTLD-tau, and DS/AD. AV1451 also labeled neuritic plaques from patients with DS/AD, but no glial tau lesions were observed (Fig. 3). No patients with TSC exhibited glial-tau lesions such as coiled bodies, tufted astrocytes, or astrocytic plaques (Figs. 1, 2, 3). Furthermore, no patients with TSC had amyloid-beta plaques (Fig. 4). The percentage of patients from each cohort positive for AT8, GT38, and AV1451 in the studied brain regions is sunmmarized in Table 2.

\section{Semi-quantitative immunohistochemistry quantification}

Semi-quantitative analysis was utilized to provide more formal assessment of qualitative findings. For AT8 (Fig. 1B), we observed that control patients and patients

Table 1 Demographic and clinical characteristics of the study cohort by diagnosis

\begin{tabular}{|c|c|c|c|c|}
\hline & Control $(\mathrm{N}=11)$ & $D S / A D(N=9)^{a}$ & $\mathrm{TSC}(\mathrm{N}=10)$ & $\mathrm{FTD}(\mathrm{N}=5)^{\mathrm{b}}$ \\
\hline Age (Mean, SD) & $52(9)$ & $53(6)$ & $49(6)$ & $69(4)$ \\
\hline \multicolumn{5}{|l|}{ Race (N, percent) } \\
\hline Black & $1(9 \%)$ & $1(11 \%)$ & $0(0 \%)$ & $0(0 \%)$ \\
\hline White & $8(73 \%)$ & $8(89 \%)$ & $10(100 \%)$ & $4(80 \%)$ \\
\hline Unable to determine & $2(18 \%)$ & $0(0 \%)$ & $0(0 \%)$ & $1(20 \%)$ \\
\hline Sex (percent female) & $46 \%$ & $56 \%$ & $50 \%$ & $80 \%$ \\
\hline \multicolumn{5}{|l|}{ Epilepsy diagnosis (N, Percent) } \\
\hline Epilepsy present & $0(0 \%)$ & $2(22 \%)$ & $5(50 \%)$ & $0(0 \%)$ \\
\hline Epilepsy absent & $11(100 \%)$ & $6(67 \%)$ & $3(30 \%)$ & $5(100 \%)$ \\
\hline Unable to Determine & $0(0 \%)$ & $1(11 \%)$ & $2(20 \%)$ & $0(0 \%)$ \\
\hline IDD diagnosis (N, Percent) & $0(0 \%)$ & & & \\
\hline IDD Present & $0(0 \%)$ & $0(0 \%)$ & $5(55 \%)$ & $0(0 \%)$ \\
\hline IDD Absent & $11(100 \%)$ & $8(89 \%)$ & $3(33 \%)$ & $5(100 \%)$ \\
\hline Unable to determine & $0(0 \%)$ & $1(11 \%)$ & $2(22 \%)$ & $0(0 \%)$ \\
\hline
\end{tabular}

IDD Intellectual or developmental disability

${ }^{\text {a }}$ Three subjects carried a clinical diagnosis of Down Syndrome

${ }^{\mathrm{b}}$ Four subjects had progressive supranuclear palsy (PSP), one had FTLD-tau, unclassified 


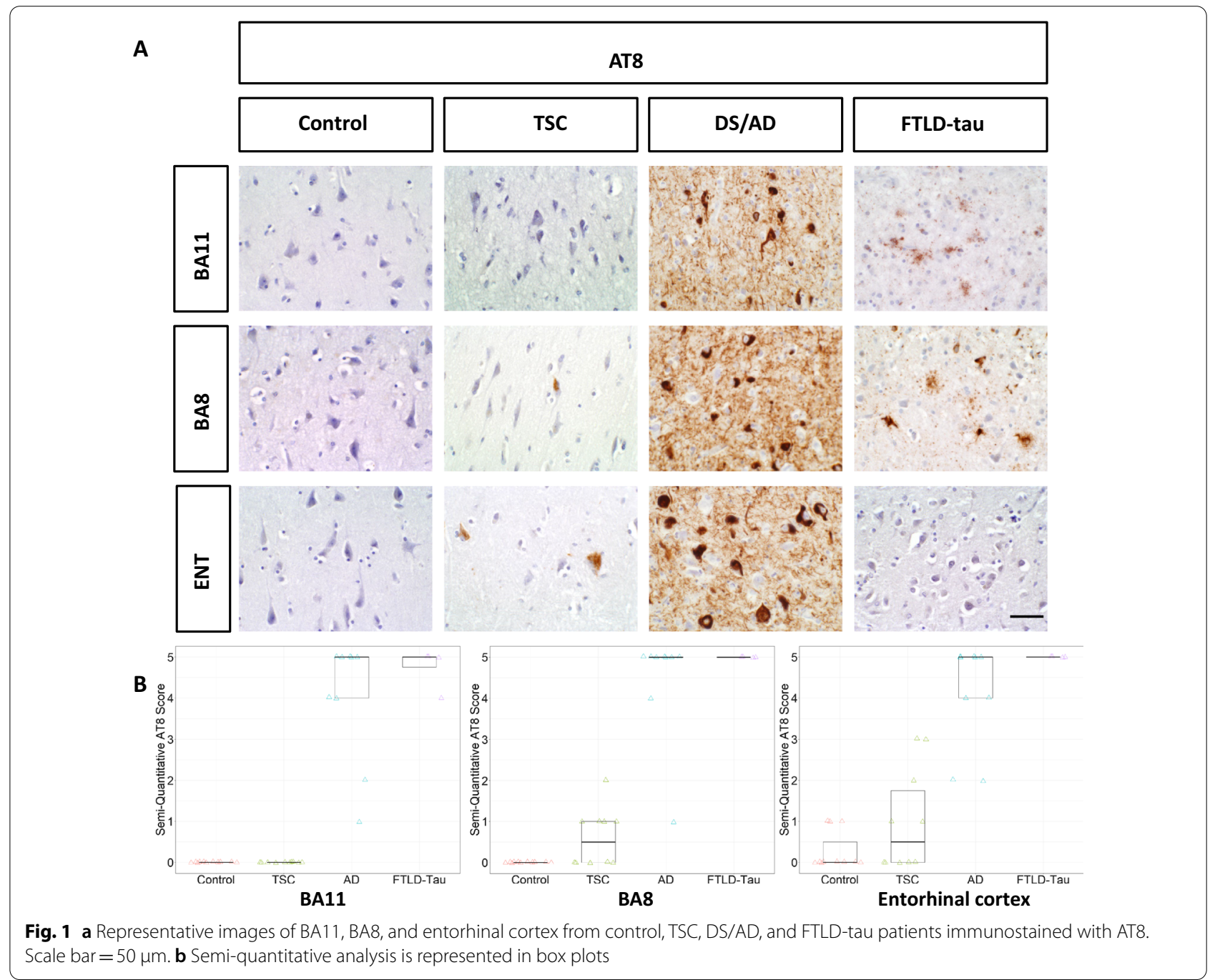

with TSC had no AT8 staining in BA11, whereas the median scores for patients with DS/AD and FTLD respectively were 5 (>10 tangles $/ 1 \mathrm{~mm}^{2}$ field). In BA8, however, patients with TSC had a median score of 0.5 ( $<1$ tangle $/ 1 \mathrm{~mm}^{2}$ field, but tangles present), compared to controls which lacked AT8 staining. The median AT8 score for patients with TSC was also 0.5 in the entorhinal cortex, but the 75 th percentile score was 1.75 , as more patients had AT8 scores of 2 (1-2 tangles/ $1 \mathrm{~mm}^{2}$ field) or 3 (3-5 tangles $/ 1 \mathrm{~mm}^{2}$ field). Median AT8 scores were high in all three brain regions for patients with DS/AD and FTLD-tau.

For GT38 scoring (Fig. 2B), while the median GT38 score in BA11 for patients with TSC was 0 , the 75 th percentile score was $1\left(<1\right.$ tangle/ $1 \mathrm{~mm}^{2}$ field $)$, as several patients had scores of 1 or 2 (1-2 tangles $/ 1 \mathrm{~mm}^{2}$ field). The median GT38 score in BA8 was 0.5 , and the 75th percentile score was 1; the median GT38 score in entorhinal cortex was 0.5 , whitle the 75 th percentile score was 2 , as some patients had scores up to 4 (6-10 tangles $/ 1 \mathrm{~mm}^{2}$ field). Patients with AD and FTLD had high median GT38 scores in all three brain regions. Control patients had minimal to no appreciable GT38 scoring in all three brain regions.

Finally, AV1451 scores (Fig. 3B) also varied across groups. Patients with TSC had median scores of $0.5,1$, and 1 in BA11, BA8, and the entorhinal cortex respectively, compared to control patients with a median score of 0 in all regions. Patients with AD and FTLD-tau had median AV1451 scores greater than 2 in all three brain regions.

\section{Discussion}

The etiology of TAND behaviors remains unknown and has been hypothesized to be related to seizures, structural changes, and intellectual disability [12-14]. Our study suggests an additional neuropathological etiology. Future studies are needed to elucidate if the neuropathological 


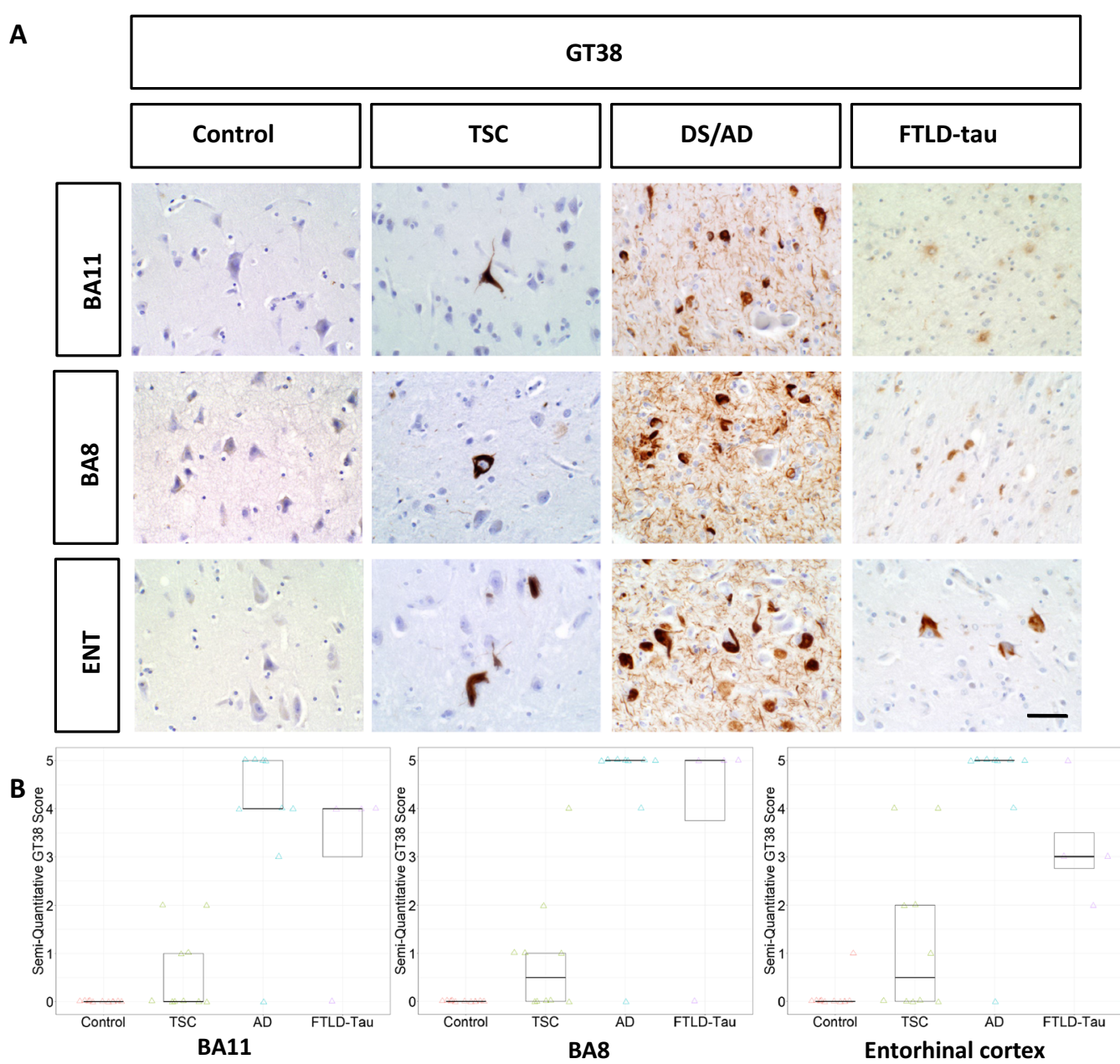

Fig. 2 a Representative images of BA11, BA8, and entorhinal cortex from control, TSC, DS/AD, and FTLD-tau patients immunostained with GT38. Scale bar $=50 \mu \mathrm{m}$. b Semi-quantitative analysis is represented in box plots

phenotypes correlate with previously described neuroradiologic phenotypes and clinically meaningful TAND clusters [11, 13].

Our study provides the first compelling evidence that TAND is a novel amyloid-independent 3R/4R tauopathy. Our finding that patients with TSC had elevated density of AT8 staining, which identifies tau phosphorylated at Ser202 and/or Thr205, compared to control patients, but less than patients with AD or FTLD suggests that phosphorylated tau accumulates to some extent in patients with TSC. Additionally, our finding that the density of GT38 positive tangles was higher in patients with TSC than control patients, and again lower than patients with $\mathrm{AD} / \mathrm{FTLD}$, suggests that TSC is a mixed $3 \mathrm{R} / 4 \mathrm{R}$ tauopathy. Patients with TSC notably lacked amyloid plaques and glial-tau lesions, distinguishing the pathology underlying TAND from other neurodegenerative conditions.
It is not entirely clear why patients with TAND had a lower density of GT38 positive tangles than FTLD patients; one possible explanation is that TSC causes a diminished extent of pathological 3R/4R tau accumulation compared with classic FTLD due to a different underlying causative mechanism. Prior research has suggested that TSC1/2 mutations, excessive mTOR signaling, and dementias may be linked mechanistically [9]. A recent case-control study likewise suggested that pTau-181 was elevated in patients with TAND and that these patients had symptoms consistent with a neurodegenerative condition [5]. Our study extends this body of literature by describing the precise underlying neuropathological phenotype of patients with TAND. In particular, the hyperactivation of mTOR signaling seen in TSC could contribute to increased tau protein aggregation and the development of $3 R / 4 R$ positive tangles. 
A
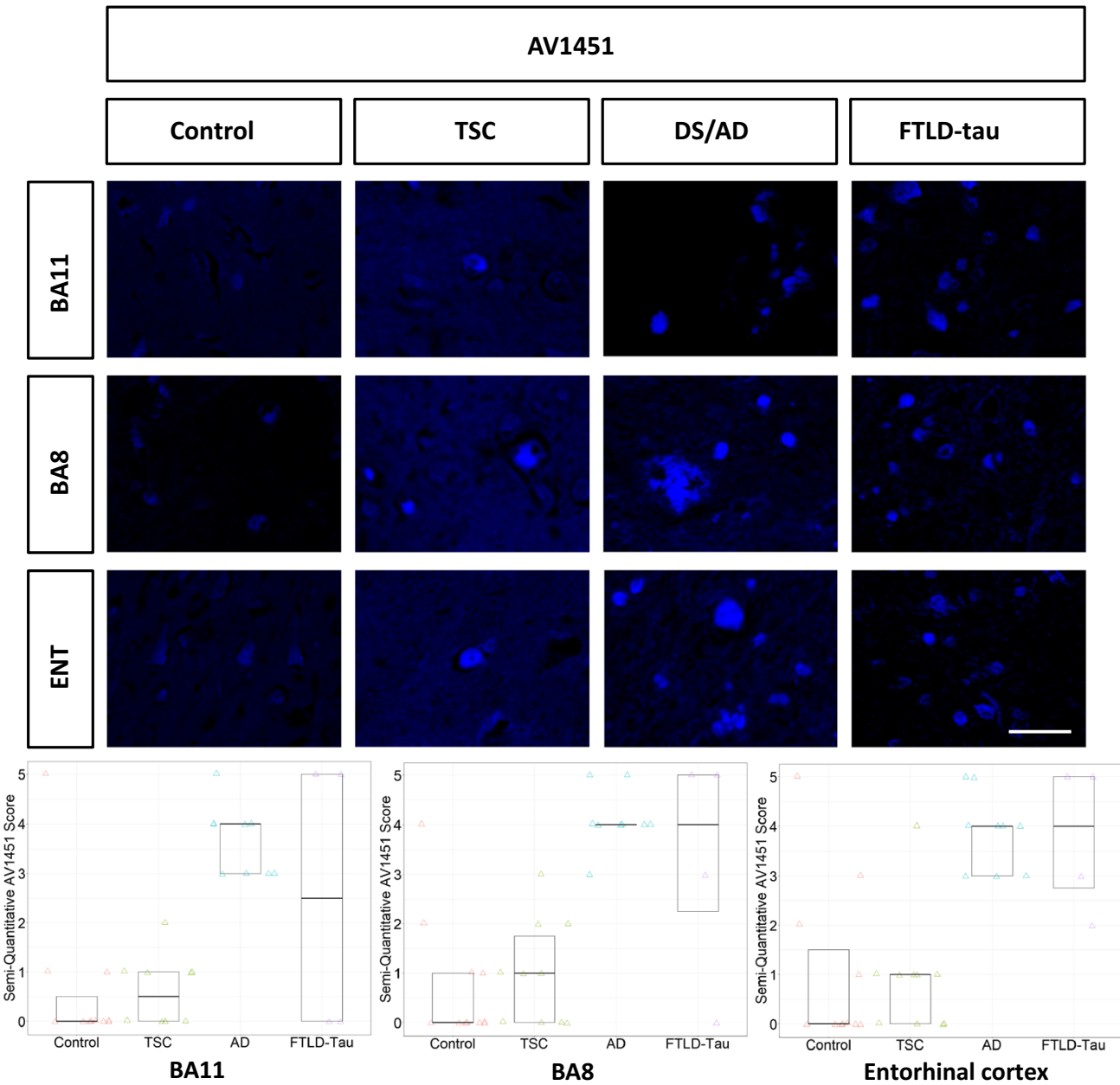

Fig. 3 a Representative images of BA11, BA8, and entorhinal cortex from control, TSC, DS/AD, and FTLD-tau patients immunostained with AV1451. Scale bar $=50 \mu \mathrm{m}$. b Semi-quantitative analysis is represented in box plots

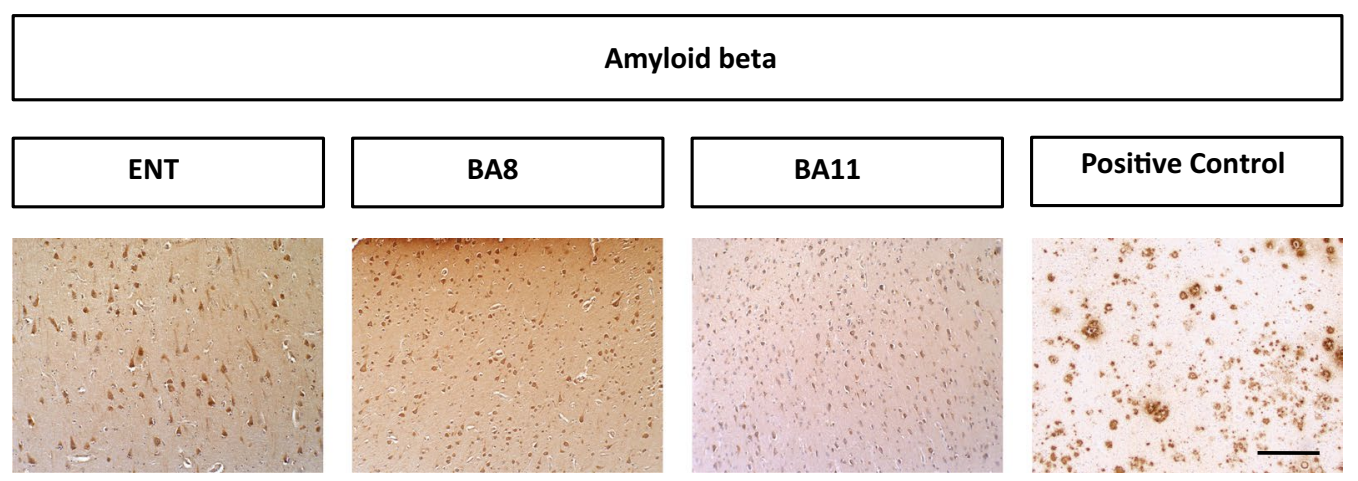

Fig. 4 Representative images of BA11, BA8, and entorhinal cortex from TSC patients immunostained with 4G8. No amyloid plaques were observed 
Table 2 Percentage of patients from each cohort positive for AT8, GT38, and AV1451 in the sampled brain regions

\begin{tabular}{llllll}
\hline & Control N=11 & DS/AD N=9 & TSC N=10 & FTLD-tau N=5 \\
\hline AT8 & Entorhinal CX & $3(27 \%)$ & $9(100 \%)$ & $5(50 \%)$ & $5(100 \%)$ \\
& BA8 & 0 & $9(100 \%)$ & $5(50 \%)$ & $5(100 \%)$ \\
GT38 & BA11 & 0 & $9(100 \%)$ & 0 & $4(80 \%)$ \\
& Entorhinal CX & $1(9 \%)$ & $8(90 \%)$ & $5(50 \%)$ & $4(80 \%)$ \\
AV1451 & BA8 & 0 & $8(90 \%)$ & $5(50 \%)$ & $4(80 \%)$ \\
& BA11 & 0 & $8(90 \%)$ & $4(40 \%)$ & $4(80 \%)$ \\
& Entorhinal CX & $3(27 \%)$ & $9(100 \%)$ & $6(60 \%)$ & $5(100 \%)$ \\
& BA8 & $3(27 \%)$ & $9(100 \%)$ & $6(60 \%)$ & $3(80 \%)$ \\
\hline
\end{tabular}

This finding, if true, would have broad implications for research into other neurodegenerative conditions, as it would imply that mTOR signaling pathway could be causally related to the formation of neurofibrillary tangles, but not neuritic plaques [3, 7]. Additional characterization between pTau distribution (and density) and clinical phenotypes has major implications both for the diagnosis and management of TAND in patients with TSC and for the pathogenesis of 3R/4R tau commonly seen in Alzheimer's disease.

Another amyloid independent 3R/4R tauopathy named Primary Age Related Tauopathy (PART) was considered to explain the findings in the TSC cohort. What makes PART less likely is the age at which the pTau was found. The mean age of the TSC cohort was 49 (Table 1 ). In an autopsy study, $20-25 \%$ of patients age 90 or older were found to have PART [2]. More importantly, PART is defined by the accumulation of $3 R / 4 R$ pTau in the mesial temporal lobes. The TSC cohort from this study displayed 3R/4R pTau in both the frontal and temporal lobes. For these reasons, PART is unlikely to explain our findings in the TSC cohort. Future research is needed to determine the precise mechanism through which mTOR signaling pathway may be related to the development of neurofibrillary tangles.

Strengths of this study include its comparison to age and gender matched healthy controls, as well as its simultaneous evaluation of multiple neuropathologies, including AD, TSC, FTLD-tau, and other neurodegenerative disorders. Additional strengths include detailed, semi-quantitative neuropathological evaluation of various tau isoforms, allowing for precise definition of the specific tauopathy seen in patients with TAND.

This study has limitations. First and foremost, its sample size was small, as TAND is a rare condition, which prevented us from utilizing formal tests of statistical significance for the associations observed. Additionally, this study was unable to control for intellectual ability/cognitive status, which has been suggested to be a major risk factor for the clinical presentation of TAND [12]. Despite these limitations, this study suggests that TAND is a novel $3 R / 4 R$ tauopathy independent of amyloid plaque formation, and points to promising new directions for research into the mechanism of TAND symptoms and novel anti-tau therapies in $\mathrm{AD}$.

\section{Acknowledgements}

We would like to thank Avid Radiopharmacheuticals for providing the AV1451 radioligand for this study. We would like to thank the NIH NeuroBiobank for supplying the brain tissue. Lastly, we thank Nikki Steinsiek for her input on this paper.

\section{Authors' contributions}

AJL substantially contributed to the conception, design, analysis, interpretation and draft of this work. JBL substantially contributed to the acquisition, interpretation and draft of this work. JE made substantial contributions to the acquisition and interpretation of this work. JB substantially contributed to the conception, design, analysis, interpretation of data and draft of this work. RO made substantial contributions to the analysis, interpretation and draft of this work. SHW made substantial contributions to the conception, acquisition, analysis, interpretation and draft of this work.

\section{Funding}

Funding for this project was provided by the Duke University, Department of Neurology.

\section{Availability of data and materials}

Data sharing not applicable to this article as no datasets were generated or analysed during the current study.

\section{Declarations}

Ethics approval and consent to participate

Duke University IRB approved protocol (protocol ID: Pro00105057) was completed prior to obtaining brain tissue from the $\mathrm{NIH}$ biobank.

\section{Consent for publication}

Not applicable.

\section{Competing interests}

The authors declare that they have no competing interests.

Received: 9 February 2022 Accepted: 11 February 2022

Published online: 03 March 2022 


\section{References}

1. Baker SL, Harrison TM, Maass A, La JR, Jagust WJ (2019) Effect of off-target binding on 18F-flortaucipir variability in healthy controls across the life span. J Nucl Med 60:1444-1451. https://doi.org/10.2967/jnumed.118. 224113

2. Bell WR, An Y, Kageyama Y, English C, Rudow GL, Pletnikova O, Thambisetty M, O'Brien R, Moghekar AR, Albert MS, Rabins PV, Resnick SM, Troncoso JC (2019) Neuropathologic, genetic, and longitudinal cognitive profiles in primary age-related tauopathy (PART) and Alzheimer's disease. Alzheimers Dement J Alzheimers Assoc 15:8-16. https://doi.org/10. 1016/j.jalz.2018.07.215

3. Cai Z, Chen G, He W, Xiao M, Yan L-J (2015) Activation of mTOR: a culprit of Alzheimer's disease? Neuropsychiatr Dis Treat 11:1015-1030. https://doi. org/10.2147/NDT.S75717

4. Iqubal K, Liu F, Gong CXG-II (2010) Tau in Alzheimer disease and related tauopathies. Curr Alzheimers Res 7:656-664. https://doi.org/10.2174/ 156720510793611592

5. Liu AJ, Staffaroni AM, Rojas-Martinez JC, Olney NT, Alquezar-Burillo C, Ljubenkov PA, La Joie R, Fong JC, Taylor J, Karydas A, Ramos EM, Coppola G, Boxer AL, Rabinovici GD, Miller BL, Kao AW (2019) Association of cognitive and behavioral features between adults with tuberous sclerosis and frontotemporal dementia. JAMA Neurol. https://doi.org/10.1001/jaman eurol.2019.4284

6. Mattsson N, Schöll M, Strandberg O, Smith R, Palmqvist S, Insel PS, Hägerström D, Ohlsson T, Zetterberg H, Jögi J, Blennow K, Hansson O (2017) 18 F-AV-1451 and CSF T-tau and P-tau as biomarkers in Alzheimer's disease. EMBO Mol Med 9:1212-1223. https://doi.org/10.15252/emmm.20170 7809

7. Mueed Z, Tandon P, Maurya SK, Deval R, Kamal MA, Poddar NK (2019) Tau and mTOR: the hotspots for multifarious diseases in Alzheimer's development. Front Neurosci 12:1017. https://doi.org/10.3389/fnins.2018.01017

8. Northrup H, Krueger DA, Roberds S, Smith K, Sampson J, Korf B, Kwiatkowski DJ, Mowat D, Nellist M, Povey S, de Vries P, Byars A, Dunn D, Ess K, Hook D, Jansen A, King B, Sahin M, Whittemore V, Thiele E, Bebin EM, Chugani HT, Crino P, Curatolo P, Holmes G, Nabbout R, O'Callaghan F, Wheless J, Wu J, Darling TN, Cowen EW, Gosnell E, Hebert A, Mlynarczyk G, Soltani K, Teng J, Wataya-Kaneda M, Witman PM, Kingswood C, Bissler J, Budde K, Hulbert J, Guay-Woodford L, Sauter M, Zonneberg B, Jóźwiak S, Bartels U, Berhouma M, Franz DN, Koenig MK, Roach ES, Roth J, Wang H, Weiner H, McCormack FX, Almoosa K, Brody A, Burger C, Cottin V, Finlay G, Glass J, Henske EP, Johnson S, Kotloff R, Lynch D, Moss J, Rhu J, Da Silva AT, Young LR, Knilans T, Hinton R, Prakash A, Romp R, Singh AD, Debroy A, Chen PL, Sparagana S, Frost MD (2013) Tuberous sclerosis complex diagnostic criteria update: Recommendations of the 2012 international tuberous sclerosis complex consensus conference. Pediatr Neurol 49:243-254. https://doi.org/10.1016/j.pediatrneurol.2013.08.001

9. Olney NT, Alquezar C, Ramos EM, Nana AL, Fong JC, Karydas AM, Taylor JB, Stephens ML, Argouarch AR, Van Berlo VA, Dokuru DR, Sherr EH, Jicha GA, Dillon WP, Desikan RS, De May M, Seeley WW, Coppola G, Miller BL, Kao AW (2017) Linking tuberous sclerosis complex, excessive mTOR signaling, and age-related neurodegeneration: a new association between TSC 1 mutation and frontotemporal dementia. Acta Neuropathol (Berl) 134:813-816. https://doi.org/10.1007/s00401-017-1764-0

10. Sarnat HB, Flores-Sarnat L (2015) Infantile tauopathies: Hemimegalencephaly; tuberous sclerosis complex; focal cortical dysplasia 2; ganglioglioma. Brain Dev 37:553-562. https://doi.org/10.1016/j.braindev.2014 08.010

11. Sidira C, Vargiami E, Anastasiou A, Talimtzi P, Kyriazi M, Dragoumi P, Spanou M, Ntinopoulos A, Dalpa E, Evangeliou A, Zafeiriou DI (2021) The complex interplay of cortex, cerebellum, and age in a cohort of pediatric patients with tuberous sclerosis complex. Pediatr Neurol 123:43-49. https://doi.org/10.1016/j.pediatrneurol.2021.06.009

12. de Vries PJ, Belousova E, Benedik MP, Carter T, Cottin V, Curatolo P, Dahlin $M, D$ 'Amato L, Beaure d'Augères G, Ferreira JC, Feucht M, Fladrowski C, Hertzberg C, Jozwiak S, Lawson JA, Macaya A, Marques R, Nabbout R, O'Callaghan F, Qin J, Sander V, Sauter M, Shah S, Takahashi Y, Touraine R, Youroukos S, Zonnenberg B, Kingswood JC, Jansen AC, Investigators T, Shinohara N, Horie S, Kubota M, Tohyama J, Imai K, Kaneda M, Kaneko H, Uchida Y, Kirino T, Endo S, Inoue Y, Uruno K, Serdaroglu A, Yapici Z, Anlar B, Altunbasak S, Lvova O, Belyaev OV, Agranovich O, Levitina EV, Maksimova YV, Karas A, Jiang Y, Zou L, Xu K, Zhang Y, Luan G, Zhang Y, Wang Y, Jin
M, Ye D, Liao W, Zhou L, Liu J, Liao J, Yan B, Deng Y, Jiang L, Liu Z, Huang S, Li H, Kim K, Chen P-L, Lee H-F, Tsai J-D, Chi C-S, Huang C-C, Riney K, Yates D, Kwan P, Likasitwattanakul S, Nabangchang C, Chomtho LTK, Katanyuwong K, Sriudomkajorn S, Wilmshurst J, Segel R, Gilboa T, Tzadok M, Fattal-Valevski A, Papathanasopoulos P, Papavasiliou AS, Giannakodimos S, Gatzonis S, Pavlou E, Tzoufi M, Vergeer AMH, Dhooghe M, Verhelst $H$, Roelens F, Nassogne MC, Defresne P, Waele LD, Leroy P, Demonceau $N$, Legros B, Bogaert PV, Ceulemans B, Dom L, Castelnau P, Martin ADS, Riquet A, Milh M, Cances C, Pedespan J-M, Ville D, Roubertie A, Auvin S, Berquin P, Richelme C, Allaire C, Gueden S, Tich SNT, Godet B, Rojas MLRF, Planas JC, Bermejo AM, Dura PS, Aparicio SR, Gonzalez MJM, Pison JL, Barca MOB, Laso EL, Luengo OA, Rodriguez FJA, Dieguez IM, Salas AC, Carrera IM, Salcedo EM, Petri MEY, Candela RC, da Carrilho IC, Vieira JP, da Monteiro JPSO, de Leao MJSOF, Luis CSMR, Mendonca CP, Endziniene M, Strautmanis J, Talvik I, Canevini MP, Gambardella A, Pruna D, Buono S, Fontana E, Bernardina BD, Burloiu C, Cosma ISB, Vintan MA, Popescu L, Zitterbart K, Payerova J, Bratsky L, Zilinska Z, Gruber-Sedlmayr U, Baumann M, Haberlandt E, Rostasy K, Pataraia E, Elmslie F, Johnston CA, Crawford P, Uldall P, Uvebrant P, Rask O, Bjoernvold M, Brodtkorb E, Sloerdahl A, Solhoff R, Jaatun MSG, Mandera M, Radzikowska EJ, Wysocki M, Fischereder M, Kurlemann G, Wilken B, Wiemer-Kruel A, Budde K, Marquard K, Knuf M, Hahn A, Hartmann H, Merkenschlager A, Trollmann R (2020) Tuberous sclerosis complex-associated neuropsychiatric disorders (TAND): new findings on age, sex, and genotype in relation to intellectual phenotype. Front Neurol. https://doi.org/10.3389/fneur.2020.00603

13. de Vries PJ, Belousova E, Benedik MP, Carter T, Cottin V, Curatolo P, D'Amato L, Beure d'Augères G, Ferreira JC, Feucht M, Fladrowski C, Hertzberg C, Jozwiak S, Lawson JA, Macaya A, Marques R, Nabbout R, O'Callaghan F, Qin J, Sander V, Sauter M, Shah S, Takahashi Y, Touraine R, Youroukos S, Zonnenberg B, Kingswood JC, Jansen AC, TOSCA Consortium and TOSCA Investigators (2020) Natural clusters of tuberous sclerosis complex (TSC)-associated neuropsychiatric disorders (TAND): new findings from the TOSCA TAND research project. J Neurodev Disord 12:24. https://doi.org/10.1186/s11689-020-09327-0

14. De Vries PJ, Whittemore VH, Leclezio L, Byars AW, Dunn D, Ess KC, Hook D, King BH, Sahin M, Jansen A (2015) Tuberous sclerosis associated neuropsychiatric disorders (TAND) and the TAND checklist. Pediatr Neurol 52:25-35. https://doi.org/10.1016/j.pediatrneurol.2014.10.004

\section{Publisher's Note}

Springer Nature remains neutral with regard to jurisdictional claims in published maps and institutional affiliations.
Ready to submit your research? Choose BMC and benefit from:

- fast, convenient online submission

- thorough peer review by experienced researchers in your field

- rapid publication on acceptance

- support for research data, including large and complex data types

- gold Open Access which fosters wider collaboration and increased citations

- maximum visibility for your research: over 100M website views per year

At BMC, research is always in progress.

Learn more biomedcentral.com/submissions 